\title{
Extragradient subgradient methods for solving bilevel equilibrium problems
}

\author{
Tadchai Yuying ${ }^{1}$, Bui Van Dinh², Do Sang Kim³ and Somyot Plubtieng ${ }^{1,4^{*}}$
}

\author{
"Correspondence: \\ somyotp@nu.ac.th \\ 'Department of Mathematics, \\ Faculty of Science, Naresuan \\ University, Phitsanulok, Thailand \\ ${ }^{4}$ Center of Excellence in Nonlinear \\ Analysis and Optimization, Faculty \\ of Science, Naresuan University, \\ Phitsanulok, Thailand \\ Full list of author information is \\ available at the end of the article
}

\begin{abstract}
In this paper, we propose two algorithms for finding the solution of a bilevel equilibrium problem in a real Hilbert space. Under some sufficient assumptions on the bifunctions involving pseudomonotone and Lipschitz-type conditions, we obtain the strong convergence of the iterative sequence generated by the first algorithm. Furthermore, the strong convergence of the sequence generated by the second algorithm is obtained without a Lipschitz-type condition.
\end{abstract}

MSC: 47J25; 65K10; 65K15; 90C25

Keywords: Bilevel equilibrium problems; Extragradient Subgradient-Halpern methods; Armijo line search; Strong convergence

\section{Introduction}

Let $C$ be a nonempty closed convex subset of a real Hilbert space $H$, and let $f$ and $g$ be bifunctions from $H \times H$ to $\mathbb{R}$ such that $f(x, x)=0$ and $g(x, x)=0$ for all $x \in H$. The equilibrium problem associated with $g$ and $C$ is denoted by $E P(C, g)$ : Find $x^{*} \in C$ such that

$$
g\left(x^{*}, y\right) \leq 0 \quad \text { for every } y \in C \text {. }
$$

The solution set of problem (1) is denoted by $\Omega$. The equilibrium problem is very important because many problems arise in applied areas such as the fixed point problem, the (generalized) Nash equilibrium problem in game theory, the saddle point problem, the variational inequality problem, the optimization problem and others.

The basic method for solving the monotone equilibrium problem is the proximal method (see [14, 16, 19]). In 2008, Tran et al. [25] proposed the extragradient algorithm for solving the equilibrium problem by using the strongly convex minimization problem to solve at each iteration. Furthermore, Hieu [9] introduced subgradient extragradient methods for pseudomonotone equilibrium problem and the other methods (see the details in $[1,8,10,15,17,22,28])$.

In this paper, we consider the equilibrium problem whose constraints are the solution sets of equilibrium problems (bilevel equilibrium problems) : Find $x^{*} \in \Omega$ such that

$$
f\left(x^{*}, y\right) \geq 0 \quad \text { for every } y \in \Omega .
$$

(c) The Author(s) 2018. This article is distributed under the terms of the Creative Commons Attribution 4.0 International License (http://creativecommons.org/licenses/by/4.0/), which permits unrestricted use, distribution, and reproduction in any medium, provided you give appropriate credit to the original author(s) and the source, provide a link to the Creative Commons license, and indicate if changes were made. 
The solution set of problem (2) is denoted by $\Omega^{*}$. The bilevel equilibrium problems were introduced by Chadli et al. [4] in 2000. This kind of problems is very important and interesting because it is a generalization class of problems such as optimization problems over equilibrium constraints, variational inequality over equilibrium constraints, hierarchical minimization problems, and complementarity problems. Furthermore, the particular case of the bilevel equilibrium can be applied to a real word model such as the variational inequality over the fixed point set of a firmly nonexpansive mapping applied to the power control problem of CDMA networks which were introduced by liduka [11]. For more on the relation of bilevel equilibrium with particular cases, see [7, 12, 21].

Methods for solving bilevel equilibrium problems have been studied extensively by many authors. In 2010, Moudafi [20] introduced a simple proximal method and proved the weak convergence to a solution of problem (2). In 2014, Quy [23] introduced the algorithm by combining the proximal method with the Halpern method for solving bilevel monotone equilibrium and fixed point problem. For more details and most recent works on the methods for solving bilevel equilibrium problems, we refer the reader to [2, 5, 24]. The authors considered the method for monotone and pseudoparamonotone equilibrium problem. If a bifunction is more generally monotone, we cannot use the above methods for solving bilevel equilibrium problem, for example, the pseudomonotone property.

Inspired by the above work, in this paper, we propose a method for finding the solution for bilevel equilibrium problems where $f$ is strongly monotone and $g$ is pseudomonotone and Lipschitz-type continuous. Firstly, we obtain the convergent sequence by combining an extragradient subgradient method with the Halpern method. Second, we obtain the convergent sequence without Lipschitz-type continuity on bifunction $g$ by combining an Armijo line search with the extragradient subgradient method.

\section{Preliminaries}

Let $C$ be a nonempty closed convex subset of a real Hilbert space $H$. Denote that $x_{n} \rightarrow x$ and $x_{n} \rightarrow x$ are the weak convergence and the strong convergence of a sequence $\left\{x_{n}\right\}$ to $x$, respectively. For every $x \in H$, there exists a unique element $P_{C} x$ defined by

$$
P_{C} x=\inf \{\|x-y\|: y \in C\} .
$$

It is also known that $P_{C}$ is a nonexpansive mapping from $H$ onto $C$, i.e., $\left\|P_{C}(x)-P_{C}(y)\right\| \leq$ $\|x-y\| \forall x, y \in H$. For every $x \in H$ and $y \in C$, we have

$$
\|x-y\|^{2} \geq\left\|x-P_{C} x\right\|^{2}+\left\|y-P_{C} x\right\|^{2} \quad \text { and } \quad\left\langle x-P_{C} x, P_{C} x-y\right\rangle \geq 0 .
$$

A bifunction $\psi: H \times H \rightarrow \mathbb{R}$ is called:

(i) $\beta$-strongly monotone on $C$ if

$$
\psi(x, y)+\psi(y, x) \leq-\beta\|x-y\|^{2} \quad \forall x, y \in C
$$

(ii) monotone on $C$ if

$$
\psi(x, y)+\psi(y, x) \leq 0 \quad \forall x, y \in C ;
$$


(iii) pseudomonotone on $C$ if

$$
\psi(x, y) \geq 0 \Rightarrow \psi(y, x) \leq 0, \quad \forall x, y \in C
$$

It is easy to check that the monotone bifunction implies the pseudomonotone bifunction. On the other hand, if the bifunction is pseudomonotone, then we cannot guarantee that the bifunction is monotone, for example, let $\phi(x, y)=\frac{2 y-x}{1-x}$ for all $x, y \in \mathbb{R}$. It follows that $\phi$ is pseudomonotone on $\mathbb{R}^{+} \backslash\{0\}$ but $\phi$ is not monotone on $\mathbb{R}^{+} \backslash\{0\}$. Let $\psi(x, \cdot)$ be convex for every $x \in H$. For each fixed $x \in H$, the subdifferential of $\psi(x,$.$) at x$, denoted by $\partial_{2} \psi(x, x)$, is defined by

$$
\begin{aligned}
\partial_{2} \psi(x, x) & =\{w \in H: \psi(x, y)-\psi(x, x) \geq\langle w, y-x\rangle \forall y \in H\} \\
& =\{w \in H: \psi(x, y) \geq\langle w, y-x\rangle \forall y \in H\},
\end{aligned}
$$

studied in [13]. In this paper, we consider the bifunctions $f$ and $g$ under the following conditions.

\section{Condition A}

(A1) $f(x, \cdot)$ is convex, weakly lower semicontinuous and subdifferentiable on $H$ for every fixed $x \in H$.

(A2) $f(\cdot, y)$ is weakly upper semicontinuous on $H$ for every fixed $y \in H$.

(A3) $f$ is $\beta$-strongly monotone on $H$.

(A4) For each $x, y \in H$, there exists $L>0$ such that

$$
\|w-v\| \leq L\|x-y\|, \quad \forall w \in \partial_{2} f(x, x), v \in \partial_{2} f(y, y) .
$$

(A5) The function $x \mapsto \partial_{2} f(x, x)$ is bounded on the bounded subsets of $H$.

\section{Condition B}

(B1) $g(x, \cdot)$ is convex, weakly lower semicontinuous, and subdifferentiable on $H$ for every fixed $x \in H$.

(B2) $g(\cdot, y)$ is weakly upper semicontinuous on $H$ for every fixed $y \in H$.

(B3) $g$ is pseudomonotone on $C$ with respect to $\Omega$, i.e.,

$$
g\left(x, x^{*}\right) \leq 0, \quad \forall x \in C, x^{*} \in \Omega
$$

(B4) $g$ is Lipschitz-type continuous, i.e., there are two positive constants $L_{1}, L_{2}$ such that $g(x, y)+g(y, z) \geq g(x, z)-L_{1}\|x-y\|^{2}-L_{2}\|y-z\|^{2}, \forall x, y, z \in H$.

(B5) $g$ is jointly weakly continuous on $H \times H$ in the sense that, if $x, y \in H$ and $\left\{x_{n}\right\},\left\{y_{n}\right\} \in H$ converge weakly to $x$ and $y$, respectively, then $g\left(x_{n}, y_{n}\right) \rightarrow g(x, y)$ as $n \rightarrow+\infty$.

Example 2.1 Let $f, g: \mathbb{R} \times \mathbb{R} \rightarrow \mathbb{R}$ be defined by $f(x, y)=5 y^{2}-7 x^{2}+2 x y$ and $g(x, y)=$ $2 y^{2}-7 x^{2}+5 x y$. It follows that $f$ and $g$ satisfy Condition A and Condition B, respectively.

Lemma 2.2 ([3, Propositions 3.1, 3.2]) If the bifunction g satisfies Assumptions (B1), (B2), and (B3), then the solution set $\Omega$ is closed and convex. 
Remark 2.3 Let the bifunction $f$ satisfy Assumption A and the bifunction $g$ satisfy B. If $\Omega \neq$ $\emptyset$, then the bilevel equilibrium problem (2) has a unique solution, see the details in [23].

Lemma 2.4 ([6]) Let $C$ be a nonempty closed convex subset of a real Hilbert space $H$ and $\phi: C \rightarrow \mathbb{R}$ be a convex, lower semicontinuous, and subdifferentiable function on $C$. Then $x^{*}$ is a solution to the convex optimization problem $\min \{\phi(x): x \in C\}$ if and only if $0 \in$ $\partial \phi\left(x^{*}\right)+N_{C}\left(x^{*}\right)$, where $\partial \phi(\cdot)$ denotes the subdifferential of $\phi$ and $N_{C}\left(x^{*}\right)$ is the normal cone of $C$ at $x^{*}$.

Lemma 2.5 ([27]) Let $\left\{a_{n}\right\}$ be a sequence of nonnegative real numbers, $\left\{\alpha_{n}\right\}$ be a sequence in $(0,1)$, and $\left\{\xi_{n}\right\}$ be a sequence in $\mathbb{R}$ satisfying the condition

$$
a_{n+1} \leq\left(1-\alpha_{n}\right) a_{n}+\alpha_{n} \xi_{n} \quad \forall n \geq 0
$$

where $\sum_{n=0}^{\infty} \alpha_{n}=\infty$ and $\lim \sup _{n \rightarrow \infty} \xi_{n} \leq 0$. Then $\lim _{n \rightarrow \infty} a_{n}=0$.

Lemma 2.6 ([18]) Let $\left\{a_{n}\right\}$ be a sequence of real numbers that does not decrease at infinity, in the sense that there exists a subsequence $\left\{a_{n_{j}}\right\}$ of $\left\{a_{n}\right\}$ such that

$$
a_{n_{j}}<a_{n_{j+1}} \text { for all } j \geq 0 .
$$

Also consider the sequence of integers $\{\tau(n)\}_{n \geq n_{0}}$ defined, for all $n \geq n_{0}$, by

$$
\tau(n)=\max \left\{k \leq n \mid a_{k}<a_{k+1}\right\} .
$$

Then $\{\tau(n)\}_{n \geq n_{0}}$ is a nondecreasing sequence verifying

$$
\lim _{n \rightarrow \infty} \tau(n)=\infty,
$$

and, for all $n \geq n_{0}$, the following two estimates hold:

$$
a_{\tau(n)} \leq a_{\tau(n)+1} \quad \text { and } \quad a_{n} \leq a_{\tau(n)+1} .
$$

Lemma 2.7 Suppose that $f$ is $\beta$-strongly monotone on $H$ and satisfies (A4). Let $0<\alpha<1$, $0 \leq \eta \leq 1-\alpha$, and $0<\mu<\frac{2 \beta}{L^{2}}$. For each $x, y \in H, w \in \partial_{2} f(x, x)$, and $v \in \partial_{2} f(y, y)$, we have

$$
\|(1-\eta) x-\alpha \mu w-[(1-\eta) y-\alpha \mu v]\| \leq(1-\eta-\alpha \tau)\|x-y\|,
$$

where $\tau=1-\sqrt{1-\mu\left(2 \beta-\mu L^{2}\right)} \in(0,1]$.

Proof Let $x, y \in H, w \in \partial_{2} f(x, x)$, and $v \in \partial_{2} f(y, y)$. Thus

$$
\begin{aligned}
\| & (1-\eta) x-\alpha \mu w-[(1-\eta) y-\alpha \mu \nu] \| \\
\quad & \leq\|(1-\eta)(x-y)-\alpha \mu(w-v)\| \\
& =\|(1-\eta-\alpha)(x-y)+\alpha[(x-y)-\mu(w-v)]\| \\
& \leq(1-\eta-\alpha)\|x-y\|+\alpha\|(x-y)-\mu(w-v)\| .
\end{aligned}
$$


Since $f: H \times H \rightarrow \mathbb{R}$ is $\beta$-strongly monotone, $w \in \partial_{2} f(x, x)$, and $v \in \partial_{2} f(y, y)$, we have

$$
-\beta\|x-y\|^{2} \geq f(x, y)+f(y, x) \geq-\langle x-y, w-v\rangle .
$$

From (5) and (A4), we have

$$
\begin{aligned}
\|(x-y)-\mu(w-v)\|^{2} & =\|x-y\|^{2}-2 \mu\langle x-y, w-v\rangle+\mu^{2}\|w-v\|^{2} \\
& \leq\|x-y\|^{2}-2 \mu \beta\|x-y\|^{2}+\mu^{2} L^{2}\|x-y\|^{2} \\
& =\left(1-2 \mu \beta+\mu^{2} L^{2}\right)\|x-y\|^{2} .
\end{aligned}
$$

This implies that

$$
\|(x-y)-\mu(w-v)\| \leq \sqrt{1-2 \mu \beta+\mu^{2} L^{2}}\|x-y\| .
$$

By using (4) and (6), we can conclude that

$$
\begin{aligned}
& \|(1-\eta) x-\alpha \mu w-[(1-\eta) y-\alpha \mu \nu]\| \\
& \quad \leq(1-\eta-\alpha)\|x-y\|+\left(\alpha \sqrt{1-2 \mu \beta+\mu^{2} L^{2}}\right)\|x-y\| \\
& \quad=(1-\eta-\alpha \tau)\|x-y\|,
\end{aligned}
$$

where $\tau=1-\sqrt{1-\mu\left(2 \beta-\mu L^{2}\right)} \in(0,1]$.

\section{The extragradient subgradient Halpern methods}

In this section, we propose the algorithm for finding the solution of a bilevel equilibrium problem under the strong monotonicity of $f$ and the pseudomonotonicity and Lipschitztype continuous conditions on $g$.

\section{Algorithm 1}

Initialization: Choose $x_{0} \in H, 0<\mu<\frac{2 \beta}{L^{2}}$, the sequences $\left\{\alpha_{n}\right\} \subset(0,1),\left\{\eta_{n}\right\}$, and $\left\{\lambda_{n}\right\}$ are such that

$$
\left\{\begin{array}{l}
\lim _{n \rightarrow \infty} \alpha_{n}=0, \quad \sum_{n=0}^{\infty} \alpha_{n}=\infty \\
0 \leq \eta_{n} \leq 1-\alpha_{n} \quad \forall n \geq 0, \quad \lim _{n \rightarrow \infty} \eta_{n}=\eta<1 \\
0<\underline{\lambda} \leq \lambda_{n} \leq \bar{\lambda}<\min \left(\frac{1}{2 L_{1}}, \frac{1}{2 L_{2}}\right) .
\end{array}\right.
$$

Set $n=0$ and go to Step 1 .

Step 1. Compute

$$
\begin{aligned}
& y_{n}=\arg \min _{y \in C}\left\{\lambda_{n} g\left(x_{n}, y\right)+\frac{1}{2}\left\|y-x_{n}\right\|^{2}\right\}, \\
& z_{n}=\arg \min _{y \in C}\left\{\lambda_{n} g\left(y_{n}, y\right)+\frac{1}{2}\left\|y-x_{n}\right\|^{2}\right\} .
\end{aligned}
$$

Step 2. Compute $w_{n} \in \partial_{2} f\left(z_{n}, z_{n}\right)$ and

$$
x_{n+1}=\eta_{n} x_{n}+\left(1-\eta_{n}\right) z_{n}-\alpha_{n} \mu w_{n} .
$$

Set $n=n+1$ and go back to Step 1 . 
Theorem 3.1 Let bifunctions $f$ and $g$ satisfy Condition A and Condition B, respectively. Assume that $\Omega \neq \emptyset$. Then the sequence $\left\{x_{n}\right\}$ generated by Algorithm 1 converges strongly to the unique solution of the bilevel equilibrium problem (2).

Proof Under assumptions of two bifunctions $f$ and $g$, we get the unique solution of the bilevel equilibrium problem (2), denoted by $x^{*}$.

Step 1: Show that

$$
\left\|z_{n}-x^{*}\right\|^{2} \leq\left\|x_{n}-x^{*}\right\|^{2}-\left(1-2 \lambda_{n} L_{1}\right)\left\|x_{n}-y_{n}\right\|^{2}-\left(1-2 \lambda_{n} L_{2}\right)\left\|y_{n}-z_{n}\right\|^{2} .
$$

The definition of $y_{n}$ and Lemma 2.4 imply that

$$
0 \in \partial_{2}\left\{\lambda_{n} g\left(x_{n}, y\right)+\frac{1}{2}\left\|y-x_{n}\right\|^{2}\right\}\left(y_{n}\right)+N_{C}\left(y_{n}\right) .
$$

There are $w \in \partial_{2} g\left(x_{n}, y_{n}\right)$ and $\bar{w} \in N_{C}\left(y_{n}\right)$ such that

$$
\lambda_{n} w+y_{n}-x_{n}+\bar{w}=0 .
$$

Since $\bar{w} \in N_{C}\left(y_{n}\right)$, we have

$$
\left\langle\bar{w}, y-y_{n}\right\rangle \leq 0 \quad \text { for all } y \in C .
$$

By using (8) and (9), we obtain $\lambda_{n}\left\langle w, y-y_{n}\right\rangle \geq\left\langle x_{n}-y_{n}, y-y_{n}\right\rangle$ for all $y \in C$. Since $z_{n} \in C$, we have

$$
\lambda_{n}\left\langle w, z_{n}-y_{n}\right\rangle \geq\left\langle x_{n}-y_{n}, z_{n}-y_{n}\right\rangle .
$$

It follows from $w \in \partial_{2} g\left(x_{n}, y_{n}\right)$ that

$$
g\left(x_{n}, y\right)-g\left(x_{n}, y_{n}\right) \geq\left\langle w, y-y_{n}\right\rangle \quad \text { for all } y \in H .
$$

By using (10) and (11), we get

$$
\lambda_{n}\left\{g\left(x_{n}, z_{n}\right)-g\left(x_{n}, y_{n}\right)\right\} \geq\left\langle x_{n}-y_{n}, z_{n}-y_{n}\right\rangle .
$$

Similarly, the definition of $z_{n}$ implies that

$$
0 \in \partial_{2}\left\{\lambda_{n} g\left(y_{n}, y\right)+\frac{1}{2}\left\|y-x_{n}\right\|^{2}\right\}\left(z_{n}\right)+N_{C}\left(z_{n}\right) .
$$

There are $u \in \partial_{2} g\left(y_{n}, z_{n}\right)$ and $\bar{u} \in N_{C}\left(z_{n}\right)$ such that

$$
\lambda_{n} u+z_{n}-x_{n}+\bar{u}=0 .
$$

Since $\bar{u} \in N_{C}\left(z_{n}\right)$, we have

$$
\left\langle\bar{u}, y-z_{n}\right\rangle \leq 0 \quad \text { for all } y \in C .
$$


By using (13) and (14), we obtain $\lambda_{n}\left\langle u, y-z_{n}\right\rangle \geq\left\langle x_{n}-z_{n}, y-z_{n}\right\rangle$ for all $y \in C$. Since $x^{*} \in C$, we have

$$
\lambda_{n}\left\langle u, x^{*}-z_{n}\right\rangle \geq\left\langle x_{n}-z_{n}, x^{*}-z_{n}\right\rangle
$$

It follows from $u \in \partial_{2} g\left(y_{n}, z_{n}\right)$ that

$$
g\left(y_{n}, y\right)-g\left(y_{n}, z_{n}\right) \geq\left\langle u, y-z_{n}\right\rangle \quad \text { for all } y \in H \text {. }
$$

By using (15) and (16), we get

$$
\lambda_{n}\left\{g\left(y_{n}, x^{*}\right)-g\left(y_{n}, z_{n}\right)\right\} \geq\left\langle x_{n}-z_{n}, x^{*}-z_{n}\right\rangle .
$$

Since $x^{*} \in \Omega$, we have $g\left(x^{*}, y_{n}\right) \geq 0$. If follows from the pseudomonotonicity of $g$ on $C$ with respect to $\Omega$ that $g\left(y_{n}, x^{*}\right) \leq 0$. This implies that

$$
\left\langle x_{n}-z_{n}, z_{n}-x^{*}\right\rangle \geq \lambda_{n} g\left(y_{n}, z_{n}\right)
$$

Since $g$ is Lipschitz-type continuous, there exist two positive constants $L_{1}, L_{2}$ such that

$$
g\left(y_{n}, z_{n}\right) \geq g\left(x_{n}, z_{n}\right)-g\left(x_{n}, y_{n}\right)-L_{1}\left\|x_{n}-y_{n}\right\|^{2}-L_{2}\left\|y_{n}-z_{n}\right\|^{2}
$$

By using (18) and (19), we get

$$
\left\langle x_{n}-z_{n}, z_{n}-x^{*}\right\rangle \geq \lambda_{n}\left\{g\left(x_{n}, z_{n}\right)-g\left(x_{n}, y_{n}\right)\right\}-\lambda_{n} L_{1}\left\|x_{n}-y_{n}\right\|^{2}-\lambda_{n} L_{2}\left\|y_{n}-z_{n}\right\|^{2} .
$$

From (12) and the above inequality, we obtain

$$
2\left\langle x_{n}-z_{n}, z_{n}-x^{*}\right\rangle \geq 2\left\langle x_{n}-y_{n}, z_{n}-y_{n}\right\rangle-\lambda_{n} L_{1}\left\|x_{n}-y_{n}\right\|^{2}-\lambda_{n} L_{2}\left\|y_{n}-z_{n}\right\|^{2} .
$$

We know that

$$
\begin{aligned}
& 2\left\langle x_{n}-z_{n}, z_{n}-x^{*}\right\rangle=\left\|x_{n}-x^{*}\right\|^{2}-\left\|z_{n}-x_{n}\right\|^{2}-\left\|z_{n}-x^{*}\right\|^{2}, \\
& 2\left\langle x_{n}-y_{n}, z_{n}-y_{n}\right\rangle=\left\|x_{n}-y_{n}\right\|^{2}+\left\|z_{n}-y_{n}\right\|^{2}-\left\|x_{n}-z_{n}\right\|^{2} .
\end{aligned}
$$

From (20), we can conclude that

$$
\left\|z_{n}-x^{*}\right\|^{2} \leq\left\|x_{n}-x^{*}\right\|^{2}-\left(1-2 \lambda_{n} L_{1}\right)\left\|x_{n}-y_{n}\right\|^{2}-\left(1-2 \lambda_{n} L_{2}\right)\left\|y_{n}-z_{n}\right\|^{2}
$$

Step 2: The sequences $\left\{x_{n}\right\},\left\{w_{n}\right\},\left\{y_{n}\right\}$, and $\left\{z_{n}\right\}$ are bounded.

Since $0<\lambda_{n}<a$, where $a=\min \left(\frac{1}{2 L_{1}}, \frac{1}{2 L_{2}}\right)$, we have

$$
\left(1-2 \lambda_{n} L_{1}\right)>0 \quad \text { and } \quad\left(1-2 \lambda_{n} L_{2}\right)>0 .
$$

It follows from (7) and the above inequalities that

$$
\left\|z_{n}-x^{*}\right\| \leq\left\|x_{n}-x^{*}\right\| \quad \text { for all } n \in \mathbb{N} \text {. }
$$


By Lemma 2.7 and (21), we obtain

$$
\begin{aligned}
\left\|x_{n+1}-x^{*}\right\| & =\left\|\eta_{n} x_{n}+\left(1-\eta_{n}\right) z_{n}-\alpha_{n} \mu w_{n}-x^{*}+\eta_{n} x^{*}-\eta_{n} x^{*}+\alpha_{n} \mu \nu-\alpha_{n} \mu \nu\right\| \\
& =\left\|\left(1-\eta_{n}\right) z_{n}-\alpha_{n} \mu w_{n}-\left(1-\eta_{n}\right) x^{*}+\alpha_{n} \mu \nu+\eta\left(x_{n}-x^{*}\right)-\alpha_{n} \mu \nu\right\| \\
& \leq\left\|\left(1-\eta_{n}\right) z_{n}-\alpha_{n} \mu w_{n}-\left[\left(1-\eta_{n}\right) x^{*}-\alpha_{n} \mu \nu\right]\right\|+\eta_{n}\left\|x_{n}-x^{*}\right\|+\alpha_{n} \mu\|v\| \\
& \leq\left(1-\eta_{n}-\alpha_{n} \tau\right)\left\|z_{n}-x^{*}\right\|+\eta_{n}\left\|x_{n}-x^{*}\right\|+\alpha_{n} \mu\|v\| \\
& \leq\left(1-\eta_{n}-\alpha_{n} \tau\right)\left\|x_{n}-x^{*}\right\|+\eta_{n}\left\|x_{n}-x^{*}\right\|+\alpha_{n} \mu\|v\| \\
& =\left(1-\alpha_{n} \tau\right)\left\|x_{n}-x^{*}\right\|+\alpha_{n} \mu\|v\| \\
& =\left(1-\alpha_{n} \tau\right)\left\|x_{n}-x^{*}\right\|+\alpha_{n} \tau\left(\frac{\mu\|v\|}{\tau}\right)
\end{aligned}
$$

where $w_{n} \in \partial_{2} f\left(z_{n}, z_{n}\right)$ and $v \in \partial_{2} f\left(x^{*}, x^{*}\right)$. This implies that

$$
\left\|x_{n+1}-x^{*}\right\| \leq \max \left\{\left\|x_{n}-x^{*}\right\|, \frac{\mu\|v\|}{\tau}\right\} .
$$

By induction, we obtain

$$
\left\|x_{n}-x^{*}\right\| \leq \max \left\{\left\|x_{0}-x^{*}\right\|, \frac{\mu\|v\|}{\tau}\right\} .
$$

Thus the sequence $\left\{x_{n}\right\}$ is bounded. By using (21), we have $\left\{z_{n}\right\}$, and using Condition (A5), we can conclude that $\left\{w_{n}\right\}$ is also bounded.

Step 3: Show that the sequence $\left\{x_{n}\right\}$ converges strongly to $x^{*}$.

Since $x \in \Omega^{*}$, we have $f\left(x^{*}, y\right) \geq 0$ for all $y \in \Omega$. Thus $x^{*}$ is a minimum of the convex function $f\left(x^{*}, \cdot\right)$ over $\Omega$. By Lemma 2.4, we obtain $0 \in \partial_{2} f\left(x^{*}, x^{*}\right)+N_{\Omega}\left(x^{*}\right)$. Then there exists $v \in \partial_{2} f\left(x^{*}, x^{*}\right)$ such that

$$
\left\langle v, z-x^{*}\right\rangle \geq 0 \quad \text { for all } z \in \Omega
$$

Note that

$$
\|x-y\|^{2} \leq\|x\|^{2}-2\langle y, x-y\rangle \quad \text { for all } x, y \in H \text {. }
$$

From Lemma (2.7) and (24), we obtain

$$
\begin{aligned}
\left\|x_{n+1}-x^{*}\right\|^{2}= & \left\|\eta_{n} x_{n}+\left(1-\eta_{n}\right) z_{n}-\alpha_{n} \mu w_{n}-x^{*}\right\|^{2} \\
= & \|\left(1-\eta_{n}\right) z_{n}-\alpha_{n} \mu w_{n}-\left[\left(1-\eta_{n}\right) x^{*}-\alpha_{n} \mu \nu\right] \\
& +\eta_{n}\left(x_{n}-x^{*}\right)-\alpha_{n} \mu \nu \|^{2} \\
\leq & \|\left(1-\eta_{n}\right) z_{n}-\alpha_{n} \mu w_{n}-\left[\left(1-\eta_{n}\right) x^{*}-\alpha_{n} \mu \nu\right] \\
& +\eta_{n}\left(x_{n}-x^{*}\right) \|^{2}-2 \alpha_{n} \mu\left\langle v, x_{n+1}-x^{*}\right\rangle \\
\leq & \left\{\|\left(1-\eta_{n}\right) z_{n}-\alpha_{n} \mu w_{n}-\left[\left(1-\eta_{n}\right) x^{*}-\alpha_{n} \mu \nu\right]\right. \\
& \left.+\eta_{n}\left\|\left(x_{n}-x^{*}\right)\right\|\right\}^{2}-2 \alpha_{n} \mu\left\langle\nu, x_{n+1}-x^{*}\right\rangle
\end{aligned}
$$




$$
\begin{aligned}
& \leq\left[\left(1-\eta_{n}-\alpha_{n} \tau\right)\left\|z_{n}-x^{*}\right\|+\eta_{n}\left\|x_{n}-x^{*}\right\|\right]^{2}-2 \alpha_{n} \mu\left\langle v, x_{n+1}-x^{*}\right\rangle \\
& \leq\left(1-\eta_{n}-\alpha_{n} \tau\right)\left\|z_{n}-x^{*}\right\|^{2}+\eta_{n}\left\|x_{n}-x^{*}\right\|^{2}-2 \alpha_{n} \mu\left\langle v, x_{n+1}-x^{*}\right\rangle \\
& \leq\left(1-\eta_{n}-\alpha_{n} \tau\right)\left\|x_{n}-x^{*}\right\|^{2}+\eta_{n}\left\|x_{n}-x^{*}\right\|^{2}-2 \alpha_{n} \mu\left\langle v, x_{n+1}-x^{*}\right\rangle \\
& =\left(1-\alpha_{n} \tau\right)\left\|x_{n}-x^{*}\right\|^{2}+2 \alpha_{n} \mu\left\langle v, x^{*}-x_{n+1}\right\rangle .
\end{aligned}
$$

It follows that

$$
\left\|x_{n+1}-x^{*}\right\|^{2} \leq\left(1-\alpha_{n} \tau\right)\left\|x_{n}-x^{*}\right\|^{2}+2 \alpha_{n} \mu\left\langle\nu, x^{*}-x_{n+1}\right\rangle .
$$

Let us consider two cases.

Case 1: There exists $n_{0}$ such that $\left\{\left\|x_{n}-x^{*}\right\|\right\}$ is decreasing for $n \geq n_{0}$. Therefore the limit of sequence $\left\{\left\|x_{n}-x^{*}\right\|\right\}$ exists. By using (21) and (25), we obtain

$$
\begin{aligned}
0 \leq & \left\|x_{n}-x^{*}\right\|^{2}-\left\|z_{n}-x^{*}\right\|^{2} \\
\leq & -\frac{\alpha_{n} \tau}{1-\eta_{n}}\left\|z_{n}-x^{*}\right\|^{2}-\frac{2 \alpha_{n} \mu}{1-\eta_{n}}\left\langle v, x_{n+1}-x^{*}\right\rangle \\
& +\frac{1}{1-\eta_{n}}\left(\left\|x_{n}-x^{*}\right\|^{2}-\left\|x_{n+1}-x^{*}\right\|^{2}\right) .
\end{aligned}
$$

Since $\lim _{n \rightarrow \infty} \eta_{n}=\eta<1, \lim _{n \rightarrow \infty} \alpha_{n}=0$ and the limit of $\left\{\left\|x_{n}-x^{*}\right\|\right\}$ exists, we have

$$
\lim _{n \rightarrow \infty}\left(\left\|x_{n}-x^{*}\right\|^{2}-\left\|z_{n}-x^{*}\right\|^{2}\right)=0
$$

From $0<\lambda_{n}<a$ and inequality (7), we get

$$
(1-2 a)\left\|x_{n}-y_{n}\right\|^{2} \leq\left(1-2 \lambda_{n} L_{1}\right)\left\|x_{n}-y_{n}\right\|^{2} \leq\left\|x_{n}-x^{*}\right\|^{2}-\left\|z_{n}-x^{*}\right\|^{2}
$$

By using (27), we obtain $\lim _{n \rightarrow \infty}\left\|x_{n}-y_{n}\right\|=0$. Next, we show that

$$
\limsup _{n \rightarrow \infty}\left\langle v, x^{*}-x_{n+1}\right\rangle \leq 0
$$

Take a subsequence $\left\{x_{n_{k}}\right\}$ of $\left\{x_{n}\right\}$ such that

$$
\limsup _{n \rightarrow \infty}\left\langle v, x^{*}-x_{n+1}\right\rangle=\limsup _{k \rightarrow \infty}\left\langle v, x^{*}-x_{n_{k}}\right\rangle .
$$

Since $\left\{x_{n_{k}}\right\}$ is bounded, we may assume that $\left\{x_{n_{k}}\right\}$ converges weakly to some $\bar{x} \in H$. Therefore

$$
\limsup _{n \rightarrow \infty}\left\langle v, x^{*}-x_{n+1}\right\rangle=\limsup _{k \rightarrow \infty}\left\langle v, x^{*}-x_{n_{k}}\right\rangle=\left\langle v, x^{*}-\bar{x}\right\rangle
$$

Since $\lim _{n \rightarrow \infty}\left\|x_{n}-y_{n}\right\|=0$ and $x_{n_{k}} \rightarrow \bar{x}$, we have $y_{n_{k}} \rightarrow \bar{x}$. Since $C$ is closed and convex, it is also weakly closed and thus $\bar{x} \in C$. Next, we show that $\bar{x} \in \Omega$. From the definition of $\left\{y_{n}\right\}$ and Lemma 2.4, we obtain

$$
0 \in \partial_{2}\left\{\lambda_{n} g\left(x_{n}, y\right)+\frac{1}{2}\left\|x_{n}-y_{n}\right\|^{2}\right\}\left(y_{n}\right)+N_{C}\left(y_{n}\right) .
$$


There exist $\bar{w} \in N_{C}\left(y_{n}\right)$ and $w \in \partial_{2} g\left(x_{n}, y_{n}\right)$ such that

$$
\lambda_{n} w+y_{n}-x_{n}+\bar{w}=0 .
$$

Since $\bar{w} \in N_{C}\left(y_{n}\right)$, we have $\left\langle\bar{w}, y-y_{n}\right\rangle \leq 0$ for all $y \in C$. From (30), we obtain

$$
\lambda_{n}\left\langle w, y-y_{n}\right\rangle \geq\left\langle x_{n}-y_{n}, y-y_{n}\right\rangle \quad \text { for all } y \in C \text {. }
$$

Since $w \in \partial_{2} g\left(x_{n}, y_{n}\right)$, we have

$$
g\left(x_{n}, y\right)-g\left(x_{n}, y_{n}\right) \geq\left\langle w, y-y_{n}\right\rangle \quad \text { for all } y \in H
$$

Combining (31) and (32), we get

$$
\lambda_{n}\left\{g\left(x_{n}, y\right)-g\left(x_{n}, y_{n}\right)\right\} \geq\left\langle x_{n}-y_{n}, y-y_{n}\right\rangle \quad \text { for all } y \in C \text {. }
$$

Taking $n=n_{k}$ and $k \rightarrow \infty$ in (33), the assumption of $\lambda_{n}$ and (B5), we obtain $g(\bar{x}, y) \geq 0$ for all $y \in C$. This implies that $\bar{x} \in \Omega$. By inequality (23), we obtain $\left\langle v, \bar{x}-x^{*}\right\rangle \geq 0$. It follows from (29) that

$$
\limsup _{n \rightarrow \infty}\left\langle v, x^{*}-x_{n+1}\right\rangle \leq 0
$$

We can write inequality (25) in the following form:

$$
\left\|x_{n+1}-x^{*}\right\|^{2} \leq\left(1-\alpha_{n} \tau\right)\left\|x_{n}-x^{*}\right\|^{2}-\alpha_{n} \tau \xi_{n},
$$

where $\xi_{n}=\frac{2 \mu}{\tau}\left\langle v, x^{*}-x_{n+1}\right\rangle$. It follows from (34) that $\lim \sup _{n \rightarrow \infty} \xi_{n} \leq 0$. By Lemma 2.5, we can conclude that $\lim _{n \rightarrow \infty}\left\|x_{n}-x^{*}\right\|^{2}=0$. Hence $x_{n} \rightarrow x^{*}$ as $n \rightarrow \infty$.

Case 2: There exists a subsequence $\left\{x_{n_{j}}\right\}$ of $\left\{x_{n}\right\}$ such that $\left\|x_{n_{j}}-x^{*}\right\| \leq\left\|x_{n_{j}+1}-x^{*}\right\|$ for all $j \in \mathbb{N}$. By Lemma 2.6, there exists a nondecreasing sequence $\{\tau(n)\}$ of $\mathbb{N}$ such that $\lim _{n \rightarrow \infty} \tau(n)=\infty$, and for each sufficiently large $n \in \mathbb{N}$, we have

$$
\left\|x_{\tau(n)}-x^{*}\right\| \leq\left\|x_{\tau(n)+1}-x^{*}\right\| \text { and }\left\|x_{n}-x^{*}\right\| \leq\left\|x_{\tau(n)+1}-x^{*}\right\| .
$$

Combining (22) and (35), we have

$$
\begin{aligned}
\left\|x_{\tau(n)}-x^{*}\right\| & \leq\left\|x_{\tau(n)+1}-x^{*}\right\| \\
& \leq\left(1-\eta_{\tau(n)}-\alpha_{\tau(n)} \tau\right)\left\|z_{\tau(n)-x^{*}}\right\|+\eta_{\tau(n)}\left\|x_{\tau(n)}-x^{*}\right\|+\alpha_{\tau(n)} \mu\|v\| .
\end{aligned}
$$

From (21) and (36), we get

$$
0 \leq\left\|x_{\tau(n)}-x^{*}\right\|-\left\|z_{\tau(n)}-x^{*}\right\| \leq-\frac{\alpha_{\tau(n)} \tau}{1-\eta_{\tau(n)}}\left\|z_{\tau(n)}-x^{*}\right\|+\frac{\alpha_{\tau(n)} \mu}{1-\eta_{\tau(n)}}\|v\| .
$$

Since $\lim _{n \rightarrow \infty} \alpha_{n}=0, \lim _{n \rightarrow \infty} \eta_{n}=\eta<1,\left\{z_{n}\right\}$ is bounded, and (37), we have $\lim _{n \rightarrow \infty}\left(\| x_{\tau(n)}-\right.$ $\left.x^{*}\|-\| z_{\tau(n)}-x^{*} \|\right)=0$. It follows from the boundedness of $\left\{x_{n}\right\}$ and $\left\{z_{n}\right\}$ that

$$
\lim _{n \rightarrow \infty}\left(\left\|x_{\tau(n)}-x^{*}\right\|^{2}-\left\|z_{\tau(n)}-x^{*}\right\|^{2}\right)=0
$$


By using the assumption of $\left\{\lambda_{n}\right\}$, we get the following two inequalities:

$$
1-2 \lambda_{\tau(n)} L_{1}>1-2 a L_{1}>0 \text { and } 1-2 \lambda_{\tau(n)} L_{2}>1-2 a L_{2}>0 \text {. }
$$

From (7), we obtain

$$
\begin{gathered}
\left\|z_{\tau(n)}-x^{*}\right\|^{2} \leq\left\|x_{\tau(n)}-x^{*}\right\|^{2}-\left(1-2 \lambda_{\tau(n)} L_{1}\right)\left\|x_{\tau(n)}-y_{\tau(n)}\right\|^{2} \\
-\left(1-2 \lambda_{\tau(n)} L_{2}\right)\left\|y_{\tau(n)}-z_{\tau(n)}\right\|^{2} \\
\leq\left\|x_{\tau(n)}-x^{*}\right\|^{2}-\left(1-2 a L_{1}\right)\left\|x_{\tau(n)}-y_{\tau(n)}\right\|^{2} \\
-\left(1-2 a L_{2}\right)\left\|y_{\tau(n)}-z_{\tau(n)}\right\|^{2} .
\end{gathered}
$$

This implies that

$$
\begin{aligned}
0 & <\left(1-2 a L_{1}\right)\left\|x_{\tau(n)}-y_{\tau(n)}\right\|^{2}+\left(1-2 a L_{2}\right)\left\|y_{\tau(n)}-z_{\tau(n)}\right\|^{2} \\
& \leq\left\|x_{\tau(n)}-x^{*}\right\|^{2}-\left\|z_{\tau(n)}-x^{*}\right\|^{2} .
\end{aligned}
$$

It follows from (38) and the above inequality that

$$
\lim _{n \rightarrow \infty}\left\|x_{\tau(n)}-y_{\tau(n)}\right\|=0 \quad \text { and } \quad \lim _{n \rightarrow \infty}\left\|y_{\tau(n)}-z_{\tau(n)}\right\|=0
$$

Note that $\left\|x_{\tau(n)}-z_{\tau(n)}\right\| \leq\left\|x_{\tau(n)}-y_{\tau(n)}\right\|+\left\|y_{\tau(n)}-z_{\tau(n)}\right\|$. From (39), we have

$$
\lim _{n \rightarrow \infty}\left\|x_{\tau(n)}-z_{\tau(n)}\right\|=0
$$

By using the definition of $x_{n+1}$ and Lemma 2.7, we obtain

$$
\begin{aligned}
\left\|x_{\tau(n)+1}-x_{\tau(n)}\right\|= & \left\|\eta_{\tau(n)} x_{\tau(n)}+\left(1-\eta_{\tau(n)}\right) z_{\tau(n)}-\alpha_{\tau(n)} \mu t_{\tau(n)}-x_{\tau(n)}\right\| \\
= & \|\left(1-\eta_{\tau(n)}\right) z_{\tau(n)}-\alpha_{\tau(n)} \mu t_{\tau(n)} \\
& -\left[\left(1-\eta_{\tau(n)}\right) x_{\tau(n)}-\alpha_{\tau(n)} w_{\tau(n)}\right]-\alpha_{\tau(n)} w_{\tau(n)} \| \\
\leq & \|\left(1-\eta_{\tau(n)}\right) z_{\tau(n)}-\alpha_{\tau(n)} t_{\tau(n)} \\
& -\left[\left(1-\eta_{\tau(n)}\right) x_{\tau(n)}-\alpha_{\tau(n)} w_{\tau(n)}\right]\left\|+\alpha_{\tau(n)}\right\| w_{\tau(n)} \| \\
\leq & \left(1-\eta_{\tau(n)}-\alpha_{\tau(n)} \tau\right)\left\|z_{\tau(n)}-x_{\tau(n)}\right\|+\alpha_{\tau(n)}\left\|w_{\tau(n)}\right\| \\
\leq & \left\|z_{\tau(n)}-x_{\tau(n)}\right\|+\alpha_{\tau(n)}\left\|w_{\tau(n)}\right\|,
\end{aligned}
$$

where $t_{\tau(n)} \in \partial_{2} f\left(z_{\tau(n)}, z_{\tau(n)}\right)$ and $w_{\tau(n)} \in \partial_{2} f\left(x_{\tau(n)}, x_{\tau(n)}\right)$. Since $\lim _{n \rightarrow \infty} \alpha_{n}=0$, the boundedness of $\left\{w_{\tau(n)}\right\}$ and (40), we have $\lim _{n \rightarrow \infty}\left\|x_{\tau(n)+1}-x_{\tau(n)}\right\|=0$. As proved in the first case, we can conclude that

$$
\limsup _{n \rightarrow \infty}\left\langle v, x^{*}-x_{\tau(n)+1}\right\rangle=\limsup _{k \rightarrow \infty}\left\langle v, x^{*}-x_{\tau(n)}\right\rangle \leq 0 .
$$


Combining (25) and (35), we obtain

$$
\begin{aligned}
\left\|x_{\tau(n)+1}-x^{*}\right\|^{2} & \leq\left(1-\alpha_{\tau(n)} \tau\right)\left\|x_{\tau(n)}-x^{*}\right\|^{2}+2 \alpha_{\tau(n)} \mu\left\langle v, x^{*}-x_{\tau(n)+1}\right\rangle \\
& \leq\left(1-\alpha_{\tau(n)} \tau\right)\left\|x_{\tau(n)+1}-x^{*}\right\|^{2}+2 \alpha_{\tau(n)} \mu\left\langle v, x^{*}-x_{\tau(n)+1}\right\rangle .
\end{aligned}
$$

By using (35) again, we have

$$
\left\|x_{n}-x^{*}\right\|^{2} \leq\left\|x_{\tau(n)+1}-x^{*}\right\|^{2} \leq \frac{2 \mu}{\tau}\left\langle v, x^{*}-x_{\tau(n)+1}\right\rangle .
$$

From (41), we can conclude that $\lim _{\sup _{n \rightarrow \infty}}\left\|x_{n}-x^{*}\right\|^{2} \leq 0$. Hence $x_{n} \rightarrow x^{*}$ as $n \rightarrow \infty$. This completes the proof.

\section{The extragradient subgradient methods with line searches}

In this section, we introduce the algorithm for finding the solution of a bilevel equilibrium problem without the Lipschitz condition for the bifunction $g$.

\section{Algorithm 2}

Initialization: Choose $x_{0} \in C, 0<\mu<\frac{2 \beta}{L^{2}}, \rho \in(0,2), \gamma \in(0,1)$, the sequences $\left\{\lambda_{n}\right\},\left\{\xi_{n}\right\}$, and $\left\{\alpha_{n}\right\} \subset(0,1)$ such that

$$
\left\{\begin{array}{l}
\lim _{n \rightarrow \infty} \alpha_{n}=0, \quad \sum_{n=0}^{\infty} \alpha_{n}=\infty, \quad \sum_{n=0}^{\infty} \alpha_{n}^{2}<\infty, \\
\lambda_{n} \in[\underline{\lambda}, \bar{\lambda}] \subset(0, \infty), \quad \xi_{n} \in[\underline{\xi}, \bar{\xi}] \subset(0,2) .
\end{array}\right.
$$

Set $n=0$, and go to Step 2 .

Step 1. Compute

$$
y_{n}=\arg \min _{y \in C}\left\{\lambda_{n} g\left(x_{n}, y\right)+\frac{1}{2}\left\|y-x_{n}\right\|^{2}\right\} .
$$

If $y_{n}=x_{n}$, then set $u_{n}=x_{n}$ and go to Step 4. Otherwise, go to Step 2 .

Step 2. (Armijo line search rule) Find $m$ as the smallest positive integer number satisfying

$$
\left\{\begin{array}{l}
z_{n, m}=\left(1-\gamma^{m}\right) x_{n}+\gamma^{m} y_{n} \\
g\left(z_{n, m}, x_{n}\right)-g\left(z_{n, m}, y_{n}\right) \geq \frac{\rho}{2 \lambda_{n}}\left\|x_{n}-y_{n}\right\|^{2} .
\end{array}\right.
$$

Set $z_{n}=z_{n, m}$ and $\gamma_{n}=\gamma^{m}$.

Step 3. Choose $t_{n} \in \partial_{2} g\left(z_{n}, x_{n}\right)$ and compute $u_{n}=P_{C}\left(x_{n}-\xi_{n} \sigma_{n} t_{n}\right)$ where $\sigma_{n}=\frac{g\left(z_{n}, x_{n}\right)}{\left\|t_{n}\right\|^{2}}$

Step 4. Compute $w_{n} \in \partial_{2} f\left(u_{n}, u_{n}\right)$ and

$$
x_{n+1}=P_{C}\left(u_{n}-\alpha_{n} \mu w_{n}\right) .
$$

Set $n=n+1$, and go back to Step 1 .

Lemma 4.1 ([26]) Suppose that $y_{n} \neq x_{n}$ for some $n \in \mathbb{N}$. Then the line search corresponding to $x_{n}$ and $y_{n}$ (Step 2) is well defined, $g\left(z_{n}, x_{n}\right)>0$, and $0 \notin \partial_{2} g\left(z_{n}, x_{n}\right)$. 
Lemma 4.2 ([26]) Let $g: \Theta \times \Theta \rightarrow \infty$ be a bifunction satisfying conditions (B1) on C and (B5) on $\Theta$, where $\Theta$ is an open convex set containing C. Let $\bar{x}, \bar{y} \in \Theta$ and $\left\{x_{n}\right\},\left\{y_{n}\right\}$ be two sequences in $\Theta$ converging weakly to $\bar{x}, \bar{y} \in \Theta$, respectively. Then, for any $\varepsilon>0$, there exist $\eta>0$ and $n_{\varepsilon} \in \mathbb{N}$ such that

$$
\partial_{2} g\left(x_{n}, y_{n}\right) \subset \partial_{2} g(\bar{x}, \bar{y})+\frac{\varepsilon}{\eta} B
$$

for all $n \geq n_{\varepsilon}$, where $B$ denotes the closed unit ball in $H$.

Lemma 4.3 ([8]) Let the bifunction $g$ satisfy assumption (B1) on $C \times C$, (B5) on $\Theta \times \Theta$. Suppose that $\left\{x_{n}\right\}$ is a bounded sequence in $C, \rho>0$ and $\left\{y_{n}\right\}$ is a sequence such that

$$
y_{n}=\arg \min _{y \in C}\left\{g\left(x_{n}, y\right)+\frac{\rho}{2}\left\|y-x_{n}\right\|^{2}\right\} .
$$

Then $\left\{y_{n}\right\}$ is also bounded.

Theorem 4.4 Let the bifunction $f$ satisfy Condition A and g satisfy Conditions (B1)-(B3) and (B5). Assume that $\Omega \neq \emptyset$. Then the sequences $\left\{x_{n}\right\}$ generated by Algorithm 2 converge strongly to the unique solution of the bilevel equilibrium problem (2).

Proof Let $x^{*}$ be the unique solution of the bilevel equilibrium problem (2). Then we have $x^{*} \in \Omega$ and there exists $v \in \partial_{2} f\left(x^{*}, x^{*}\right)$ such that

$$
\left\langle v, z-x^{*}\right\rangle \geq 0 \quad \text { for all } z \in \Omega
$$

Step 1: Show that

$$
\left\|u_{n}-x^{*}\right\|^{2} \leq\left\|x_{n}-x^{*}\right\|^{2}-\xi_{n}\left(2-\xi_{2}\right)\left(\sigma_{n}\left\|t_{n}\right\|\right)^{2} .
$$

By the definition of $u_{n}$, we have

$$
\begin{aligned}
\left\|u_{n}-x^{*}\right\|^{2} & \leq\left\|P_{C}\left(x_{n}-\xi_{n} \sigma_{n} t_{n}\right)-P_{C}\left(x^{*}\right)\right\|^{2} \\
& \leq\left\|x_{n}-\xi_{n} \sigma_{n} t_{n}-x^{*}\right\|^{2} \\
& =\left\|x_{n}-x^{*}\right\|^{2}-2 \xi_{n} \sigma_{n}\left|t_{n}, x_{n}-x^{*}\right\rangle+\left(\xi_{n} \sigma_{n}\left\|t_{n}\right\|\right)^{2} .
\end{aligned}
$$

Since $t_{n} \in \partial_{2} g\left(z_{n}, x_{n}\right)$ and $g\left(z_{n}, \cdot\right)$ is convex on $C$, we have $g\left(z_{n}, x^{*}\right)-g\left(z_{n}, x_{n}\right) \geq\left\langle t_{n}, x^{*}-x_{n}\right\rangle$. It follows that

$$
\left\langle t_{n}, x_{n}-x^{*}\right\rangle \geq g\left(z_{n}, x_{n}\right)-g\left(z_{n}, x^{*}\right) .
$$

Since $g$ is pseudomonotone on $C$ with respect to $\Omega$, we have $g\left(z_{n}, x^{*}\right) \leq 0$. It follows from (46) and the definition of $\sigma_{n}$ that

$$
\left\langle t_{n}, x_{n}-x^{*}\right\rangle \geq g\left(z_{n}, x_{n}\right)=\sigma_{n}\left\|t_{n}\right\|^{2} .
$$


Combining (45) with (47), we obtain

$$
\begin{aligned}
\left\|u_{n}-x^{*}\right\|^{2} & \leq\left\|x_{n}-x^{*}\right\|^{2}-2 \xi_{n} \sigma_{n}\left(\sigma_{n}\left\|t_{n}\right\|^{2}\right)+\left(\xi_{n} \sigma_{n}\left\|t_{n}\right\|\right)^{2} \\
& =\left\|x_{n}-x^{*}\right\|^{2}-2 \xi_{n}\left(\sigma_{n}\left\|t_{n}\right\|\right)^{2}+\xi_{n}{ }^{2}\left(\sigma_{n}\left\|t_{n}\right\|\right)^{2} \\
& =\left\|x_{n}-x^{*}\right\|^{2}-\xi_{n}\left(2-\xi_{n}\right)\left(\sigma_{n}\left\|t_{n}\right\|\right)^{2} .
\end{aligned}
$$

Step 2: The sequences $\left\{x_{n}\right\},\left\{y_{n}\right\},\left\{u_{n}\right\}$, and $\left\{w_{n}\right\}$ are bounded.

Since $\xi_{n} \in[\underline{\xi}, \bar{\xi}] \subset(0,2)$ and $(44)$, we have

$$
\left\|u_{n}-x^{*}\right\| \leq\left\|x_{n}-x^{*}\right\|
$$

By the definition of $x_{n+1}$, we get

$$
\begin{aligned}
\left\|x_{n+1}-x^{*}\right\| & =\left\|P_{C}\left(u_{n}-\alpha_{n} \mu w_{n}\right)-P_{C}\left(x^{*}\right)\right\| \\
& \leq\left\|u_{n}-\alpha_{n} \mu w_{n}-x^{*}\right\| \\
& \leq\left\|\left(u_{n}-x^{*}\right)-\alpha_{n} \mu\left(w_{n}-v\right)-\alpha_{n} \mu v\right\| \\
& =\left\|\left(u_{n}-x^{*}\right)-\alpha_{n} \mu\left(w_{n}-v\right)\right\|+\alpha_{n} \mu\|v\| .
\end{aligned}
$$

From Lemma 2.7, (48), and (49), we can conclude that

$$
\begin{aligned}
\left\|x_{n+1}-x^{*}\right\| & =\left(1-\alpha_{n} \tau\right)\left\|u_{n}-x^{*}\right\|+\alpha_{n} \mu\|v\| \\
& \leq\left(1-\alpha_{n} \tau\right)\left\|x_{n}-x^{*}\right\|+\alpha_{n} \tau\left(\frac{\mu\|v\|}{\tau}\right) .
\end{aligned}
$$

This implies that

$$
\left\|x_{n+1}-x^{*}\right\| \leq \max \left\{\left\|x_{n}-x^{*}\right\|, \frac{\mu\|v\|}{\tau}\right\} .
$$

By induction, we obtain

$$
\left\|x_{n}-x^{*}\right\| \leq \max \left\{\left\|x_{0}-x^{*}\right\|, \frac{\mu\|v\|}{\tau}\right\} .
$$

Thus the sequence $\left\{x_{n}\right\}$ is bounded. Hence we can conclude from (48) and Lemma 4.3 that $\left\{y_{n}\right\}$ and $\left\{u_{n}\right\}$ are bounded, respectively. From condition (A4), we have $\left\{w_{n}\right\}$ is also bounded.

Step 3: We show that if there is a subsequence $\left\{x_{n_{k}}\right\}$ of $\left\{x_{n}\right\}$ converging weakly to $\bar{x}$ and $\lim _{k \rightarrow \infty}\left(\sigma_{n_{k}}\left\|t_{n_{k}}\right\|\right)=0$, then we have $\bar{x} \in \Omega$.

Firstly, we will show that $\left\{t_{n_{k}}\right\}$ is bounded. Since $\left\{z_{n}\right\}$ is bounded, there is a subsequence $\left\{z_{n_{k}}\right\}$ of $\left\{z_{n}\right\}$ converging weakly to $\bar{z}$ By using Lemma 4.2, for any $\varepsilon>0$, there exist $\eta>0$ and $k_{0}$ such that

$$
\partial_{2} g\left(z_{n_{k}}, x_{n_{k}}\right) \subset \partial_{2} g(\bar{z}, \bar{x})+\frac{\varepsilon}{\eta} B
$$


for all $k \geq k_{0}$. Since $\left\{t_{n_{k}}\right\} \in \partial_{2} g\left(z_{n_{k}}, x_{n_{k}}\right)$, we have $\left\{t_{n_{k}}\right\}$ is bounded. Next, we show that $\left\|x_{n_{k}}-y_{n_{k}}\right\| \rightarrow 0$. Without loss of generality, we can assume that $x_{n_{k}} \neq y_{n_{k}}$ for all $k \in \mathbb{N}$. By Lemma 4.1, we obtain $g\left(z_{n_{k}}, x_{n_{k}}\right)>0$ and $t_{n_{k}} \neq 0$. Since $\lim _{k \rightarrow \infty}\left(\sigma_{n_{k}}\left\|t_{n_{k}}\right\|\right)=0$ and $\left\{t_{n_{k}}\right\}$ is bounded, we have

$$
\lim _{k \rightarrow \infty} g\left(z_{n_{k}}, x_{n_{k}}\right)=\lim _{k \rightarrow \infty}\left(\sigma_{n_{k}}\left\|t_{n_{k}}\right\|\right)\left\|t_{n_{k}}\right\|=0 .
$$

It follows from the convexity of $g\left(z_{n_{k}}, \cdot\right)$ that

$$
\gamma_{n_{k}} g\left(z_{n_{k}}, y_{n_{k}}\right)+\left(1-\gamma_{n_{k}}\right) g\left(z_{n_{k}}, x_{n_{k}}\right) \geq g\left(z_{n_{k}}, z_{n_{k}}\right)=0
$$

This implies that

$$
\gamma_{n_{k}}\left[g\left(z_{n_{k}}, x_{n_{k}}\right)-g\left(z_{n_{k}}, y_{n_{k}}\right)\right] \leq g\left(z_{n_{k}}, x_{n_{k}}\right) .
$$

By the Armijo line search, we get $\frac{\rho}{2 \lambda_{n}}\left\|x_{n}-y_{n}\right\|^{2} \leq g\left(z_{n_{k}}, x_{n_{k}}\right)-g\left(z_{n_{k}}, y_{n_{k}}\right)$, and (52) implies that

$$
\frac{\rho \gamma_{n_{k}}}{2 \lambda_{n_{k}}}\left\|x_{n}-y_{n}\right\|^{2} \leq \gamma_{n_{k}}\left[g\left(z_{n_{k}}, x_{n_{k}}\right)-g\left(z_{n_{k}}, y_{n_{k}}\right)\right] \leq g\left(z_{n_{k}}, x_{n_{k}}\right) .
$$

Combining (51) with (53), we obtain

$$
\lim _{k \rightarrow \infty} \gamma_{n_{k}}\left\|x_{n}-y_{n}\right\|^{2}=0
$$

Then we consider two cases.

Case 1. $\lim \sup _{k \rightarrow \infty} \gamma_{n_{k}}>0$. There exist $\bar{\gamma}>0$ and a subsequence of $\left\{\gamma_{n_{k}}\right\}$ denoted again by $\left\{\gamma_{n_{k}}\right\}$ such that $\gamma_{n_{k}}>\bar{\gamma}$ for all $k$. So we get from (54) that

$$
\lim _{k \rightarrow \infty}\left\|x_{n_{k}}-y_{n_{k}}\right\|=0
$$

Since $x_{n_{k}} \rightarrow \bar{x}$ and (55), we have $y_{n_{k}} \rightarrow \bar{x}$. On the other hand, by the definition of $y_{n_{k}}$, we have

$$
\lambda_{n_{k}}\left\{g\left(x_{n_{k}}, y\right)-g\left(x_{n_{k}}, y_{n_{k}}\right)\right\} \geq\left\langle x_{n_{k}}-y_{n_{k}}, y-y_{n_{k}}\right\rangle \quad \text { for all } y \in C \text {. }
$$

Therefore

$$
\lambda_{n_{k}}\left\{g\left(x_{n_{k}}, y\right)-g\left(x_{n_{k}}, y_{n_{k}}\right)\right\} \geq-\left\|x_{n_{k}}-y_{n_{k}}\right\|\left\|y-y_{n_{k}}\right\| \quad \text { for all } y \in C \text {. }
$$

Letting $k \rightarrow \infty$ in the above inequality, using (55) and the jointly weak continuity of $g$, we have $g(\bar{x}, y)-g(\bar{x}, \bar{x}) \geq 0$ for all $y \in C$. So, $g(\bar{x}, y) \geq 0$ for all $y \in C$. Hence $\bar{x} \in \Omega$.

Case 2. $\lim \sup _{k \rightarrow \infty} \gamma_{n_{k}}=0$. From the boundedness of $\left\{y_{n}\right\}$, there exists $\left\{y_{n_{k}}\right\} \subseteq\left\{y_{n}\right\}$ such that $y_{n_{k}} \rightarrow \bar{y}$. Let $\left\{m_{k}\right\}$ be the sequence of the smallest non-negative integers such that $z_{n_{k}}=\left(1-\gamma^{m_{k}}\right) x_{n_{k}}+\gamma^{m_{k}} y_{n_{k}}$ and

$$
g\left(z_{n_{k}}, x_{n_{k}}\right)-g\left(z_{n_{k}}, y_{n_{k}}\right) \geq \frac{\rho}{2 \lambda_{n_{k}}}\left\|x_{n_{k}}-y_{n_{k}}\right\|^{2}
$$


Since $\gamma^{m_{k}} \rightarrow 0$, we have $m_{k}>0$. It follows from the Armijo line search that, for $m_{k}-1$, we have $\bar{z}_{n_{k}}=\left(1-\gamma^{m_{k}-1}\right) x_{n_{k}}+\gamma^{m_{k}-1} y_{n_{k}}$ and

$$
g\left(\bar{z}_{n_{k}}, x_{n_{k}}\right)-g\left(\bar{z}_{n_{k}}, y_{n_{k}}\right)<\frac{\rho}{2 \lambda_{n_{k}}}\left\|x_{n_{k}}-y_{n_{k}}\right\|^{2} .
$$

On the other hand, by the definition of $y_{n}$, we have

$$
\lambda_{n}\left\{g\left(x_{n}, y\right)-g\left(x_{n}, y_{n}\right)\right\} \geq\left\langle x_{n}-y_{n}, y-y_{n}\right\rangle \quad \text { for all } y \in C \text {. }
$$

Letting $n=n_{k}$ and $y=x_{n_{k}}$ in (58), we get

$$
-\lambda_{n_{k}} g\left(x_{n_{k}}, y_{n_{k}}\right) \geq\left\|x_{n_{k}}-y_{n_{k}}\right\|^{2}
$$

Combining (57) with (59), we obtain

$$
g\left(\bar{z}_{n_{k}}, x_{n_{k}}\right)-g\left(\bar{z}_{n_{k}}, y_{n_{k}}\right)<-\frac{\rho}{2} g\left(x_{n_{k}}, y_{n_{k}}\right)
$$

Since $x_{n_{k}} \rightarrow \bar{x}, y_{n_{k}} \rightarrow \bar{y}$, and $\gamma_{n_{k}} \rightarrow 0$, we have $\bar{z}_{n_{k}} \rightarrow \bar{x}$. From (60) and $g$ is jointly weakly continuous on $H \times H$, we get $-g(\bar{x}, \bar{y})<-\frac{\rho}{2} g(\bar{x}, \bar{y})$. Since $\rho \in(0,2)$, we have $g(\bar{x}, \bar{y}) \geq 0$. Taking $k \rightarrow \infty$ in (59), we obtain $\lim _{k \rightarrow \infty}\left\|x_{n_{k}}-y_{n_{k}}\right\|=0$. By Case 1 , it is immediate that $\bar{x} \in \Omega$.

Step 4: Show that the sequence $\left\{x_{n}\right\}$ converges strongly to $x^{*}$. By using the definition of $x_{n+1}$ and (44), we obtain

$$
\begin{aligned}
\left\|x_{n+1}-x^{*}\right\|^{2}= & \left\|P_{C}\left(u_{n}-\alpha_{n} \mu w_{n}-P_{C}\left(x^{*}\right)\right)\right\|^{2} \\
\leq & \left\|u_{n}-\alpha_{n} \mu w_{n}-x^{*}\right\|^{2} \\
= & \left\|u_{n}-x^{*}\right\|^{2}-2 \alpha_{n} \mu\left\langle w_{n}, u_{n}-x^{*}\right\rangle+\left(\alpha_{n} \mu\left\|w_{n}\right\|\right)^{2} \\
\leq & \left\|x_{n}-x^{*}\right\|^{2}-\xi_{n}\left(2-\xi_{n}\right)\left(\sigma_{n}\left\|t_{n}\right\|\right)^{2} \\
& \quad-2 \alpha_{n} \mu\left\langle w_{n}, u_{n}-x^{*}\right\rangle+\left(\alpha_{n} \mu\left\|w_{n}\right\|\right)^{2} \\
\leq & \left\|x_{n}-x^{*}\right\|^{2}-\left(\sigma_{n}\left\|t_{n}\right\|\right)^{2}-2 \alpha_{n} \mu\left\langle w_{n}, u_{n}-x^{*}\right\rangle \\
& +\left(\alpha_{n} \mu\left\|w_{n}\right\|\right)^{2} .
\end{aligned}
$$

Setting $a_{n}=\left\|x_{n}-x^{*}\right\|^{2}$. It follows from the boundedness of $\left\{w_{n}\right\}$ and $\left\{u_{n}\right\}$ that

$$
\left|\left\langle w_{n}, u_{n}-x^{*}\right\rangle\right| \leq M_{1} \quad \text { and } \quad\left\|w_{n}\right\|^{2} \leq M_{2}
$$

Combining (61), (62) with the definition of $a_{n}$, we get

$$
a_{n+1}-a_{n}+\left(\sigma_{n}\left\|t_{n}\right\|\right)^{2} \leq 2 \alpha_{n} \mu M_{1}+\alpha_{n}^{2} \mu^{2} M_{2}
$$

Let us consider two cases.

Case 1: There exists $n_{0}$ such that $\left\{a_{n}\right\}$ is decreasing for $n \geq n_{0}$. Therefore the limit of $\left\{a_{n}\right\}$ exists, denoted by $a$. It follows that $\lim _{n \rightarrow \infty}\left(a_{n+1}-a_{n}\right)=0$. From (63) and the definition of 
$\alpha_{n}$, we have

$$
\lim _{n \rightarrow \infty}\left(\sigma_{n}\left\|t_{n}\right\|\right)=0
$$

By the definitions of $x_{n+1}$ and $u_{n}$, we obtain

$$
\begin{aligned}
\left\|u_{n}-x_{n}\right\|^{2} & =\left\|P_{C}\left(x_{n}-\sigma_{n} t_{n}\right)-P_{C}\left(x_{n}\right)\right\| \\
& \leq\left\|x_{n}-\sigma_{n} t_{n}-x_{n}\right\| \\
& =\sigma_{n}\left\|t_{n}\right\| .
\end{aligned}
$$

It follows that $\lim _{n \rightarrow \infty}\left\|u_{n}-x_{n}\right\|^{2}=0$, which implies that

$$
\lim _{n \rightarrow \infty}\left\|u_{n}-x^{*}\right\|^{2}=a
$$

Since $\left\{u_{n}\right\} \subseteq C$ is bounded, there exists a subsequence $\left\{u_{n_{k}}\right\}$ of $\left\{u_{n}\right\}$ that converges weakly to some $\bar{u} \in C$ and satisfies the equality

$$
\liminf _{n \rightarrow \infty}\left\langle u_{n}-x^{*}, v\right\rangle=\lim _{k \rightarrow \infty}\left\langle u_{n_{k}}-x^{*}, v\right\rangle
$$

Since $u_{n_{k}} \rightarrow \bar{u} \in C$ and (43), we have

$$
\liminf _{n \rightarrow \infty}\left\langle u_{n}-x^{*}, v\right\rangle=\lim _{k \rightarrow \infty}\left\langle u_{n_{k}}-x^{*}, v\right\rangle=\left\langle\bar{u}-x^{*}, v\right\rangle \geq 0
$$

Since $w_{n} \in \partial_{2} f\left(u_{n}, u_{n}\right), v \in \partial_{2} f\left(x^{*}, x^{*}\right)$ and $f$ is $\beta$-strongly monotone on $C$, we have

$$
\begin{aligned}
\left\langle w_{n}, x^{*}-u_{n}\right\rangle \leq f\left(u_{n}, x^{*}\right) & \leq-\beta\left\|u_{n}-x^{*}\right\|^{2}-f\left(x^{*}, u_{n}\right) \\
& =-\beta\left\|u_{n}-x^{*}\right\|^{2}-\left\langle u_{n}-x^{*}, v\right\rangle .
\end{aligned}
$$

This implies that $\left\langle w_{n}, u_{n}-x^{*}\right\rangle \geq \beta\left\|u_{n}-x^{*}\right\|^{2}+\left\langle u_{n}-x^{*}, v\right\rangle$. Combining (66), (67) with the above inequality, we get

$$
\liminf _{n \rightarrow \infty}\left|w_{n}, u_{n}-x^{*}\right\rangle \geq \beta a
$$

Assume that $a>0$. Choose $\varepsilon=\frac{1}{2} \beta a$. There exists $n_{0}$ such that

$$
\left\langle u_{n}-x^{*}, w_{n}\right\rangle \geq \beta a-\varepsilon=\frac{1}{2} \beta a \quad \text { for all } n \geq n_{0} .
$$

It follows from (61) and the above inequality that $a_{n+1}-a_{n} \leq-\alpha_{n} \mu \beta a^{2}+\alpha_{n}^{2} \mu M_{2}$. Summing this inequality from $n_{0}$ to $n$, we obtain

$$
a_{n+1}-a_{n_{0}} \leq-\mu \beta a^{2} \sum_{k=n_{0}}^{n} \alpha_{k}+\mu M_{2} \sum_{k=n_{0}}^{n} \alpha_{n}^{2} .
$$

Since $\sum_{k=n_{0}}^{\infty} \alpha_{k}=\infty$ and $\sum_{k=n_{0}}^{\infty} \alpha_{n}^{2}<\infty$, we can conclude from (69) that $\liminf _{n \rightarrow \infty} a_{n}=$ $-\infty$, which is a contradiction. So, $a=0$, and we can conclude that $\lim _{n \rightarrow \infty}\left\|x_{n}-x^{*}\right\|^{2}=0$. 
Case 2: There exists a subsequence $\left\{a_{n_{j}}\right\}$ of $\left\{a_{n}\right\}$ such that $a_{n_{j}} \leq a_{n_{j}+1}$ for all $j \in \mathbb{N}$. Let $\{\tau(n)\}$ be a nondecreasing sequence defined in Lemma 2.6. Thus

$$
a_{\tau(n)} \leq a_{\tau(n)+1} \quad \text { and } \quad a_{n} \leq a_{\tau(n)+1} .
$$

From (63) and the definition of $\alpha_{n}$, we have

$$
\lim _{n \rightarrow \infty} \sigma_{\tau(n)}\left\|t_{\tau(n)}\right\|=0
$$

By the definition of $u_{\tau(n)}$, we obtain

$$
\begin{aligned}
\left\|u_{\tau(n)}-x_{\tau(n)}\right\|^{2} & =\left\|P_{C}\left(x_{\tau(n)}-\sigma_{\tau(n)} t_{\tau(n)}\right)-P_{C}\left(x_{\tau(n)}\right)\right\| \\
& \leq\left\|x_{\tau(n)}-\sigma_{\tau(n)} t_{\tau(n)}-x_{\tau(n)}\right\| \\
& =\sigma_{\tau(n)}\left\|t_{\tau(n)}\right\| .
\end{aligned}
$$

This implies that $\lim _{n \rightarrow \infty}\left\|u_{\tau(n)}-x_{\tau(n)}\right\|^{2}=0$. Since $\left\{x_{\tau(n)}\right\}$ is bounded, there exists a subsequence $\left\{x_{\tau(n)_{k}}\right\} \subseteq\left\{x_{\tau(n)}\right\}$ such that $x_{\tau(n)_{k}} \rightarrow \bar{x} \in C$ and thus $u_{\tau(n)_{k}} \rightarrow \bar{x} \in C$. From (71) and Step 3 of this proof, we get $\bar{x} \in \Omega$. Next, we will show that $u_{\tau(n)_{k}} \rightarrow x^{*}$. It follows from (61) that

$$
\begin{aligned}
2 \alpha_{\tau(n)_{k}} \mu\left\langle w_{\tau(n)_{k}}, u_{\tau(n)_{k}}-x^{*}\right\rangle \leq & a_{\tau(n)_{k}}-a_{\tau(n)_{k}+1}-\left(\sigma_{\tau(n)_{k}}\left\|t_{\tau(n)_{k}}\right\|\right)^{2} \\
& +\left(\alpha_{\tau(n)_{k}} \mu\left\|w_{\tau(n)_{k}}\right\|\right)^{2} \leq\left(\alpha_{\tau(n)_{k}} \mu\right)^{2} M_{2} .
\end{aligned}
$$

This implies that

$$
\left\langle w_{\tau(n)_{k}}, u_{\tau(n)_{k}}-x^{*}\right\rangle \leq \frac{\alpha_{\tau(n)_{k}} \mu M_{2}}{2} .
$$

Since $f$ is $\beta$-strongly monotone on $C$ and $w_{\tau(n)} \in \partial_{2} f\left(u_{\tau(n)_{k}}, u_{\tau(n)_{k}}\right)$, we have

$$
\left\langle x^{*}-u_{\tau(n)_{k}}, w_{\tau(n)_{k}}\right\rangle \leq f\left(u_{\tau(n)_{k}}, x^{*}\right) \leq-\beta\left\|u_{\tau(n)_{k}}-x^{*}\right\|^{2}-f\left(x^{*}, u_{\tau(n)_{k}}\right) .
$$

It follows from (73) and the above inequality that

$$
\begin{aligned}
\left\|u_{\tau(n)_{k}}-x^{*}\right\|^{2} & \leq \frac{1}{\beta}\left[\left\langle w_{\tau(n)_{k}}, u_{\tau(n)_{k}}-x^{*}\right\rangle-f\left(x^{*}, u_{\tau(n)_{k}}\right)\right] \\
& \leq \frac{1}{\beta}\left[\frac{\alpha_{\tau(n)_{k}} \mu M_{2}}{2}-f\left(x^{*}, u_{\tau(n)_{k}}\right)\right] .
\end{aligned}
$$

Taking $k \rightarrow \infty$, by using $u_{\tau(n)_{k}} \rightarrow \bar{x}$ and $\alpha_{\tau(n)_{k}} \rightarrow 0$, we get

$$
\underset{k \rightarrow \infty}{\limsup }\left\|u_{\tau(n)_{k}}-x^{*}\right\|^{2} \leq-f\left(x^{*}, \bar{x}\right) \leq 0
$$

Therefore $\lim _{k \rightarrow \infty}\left\|u_{\tau(n)_{k}}-x^{*}\right\|^{2}=0$. Then, it is easy to see that $\lim _{n \rightarrow \infty}\left\|u_{\tau(n)}-x^{*}\right\|^{2}=0$. By the definition of $x_{n+1}$, we have

$$
\begin{aligned}
\left\|x_{\tau(n)+1}-x^{*}\right\| & \leq\left\|u_{\tau(n)}-\alpha_{\tau(n)} \mu w_{\tau(n)}-x^{*}\right\| \\
& \leq\left\|u_{\tau(n)}-x^{*}\right\|+\alpha_{\tau(n)} \mu\left\|w_{\tau(n)}\right\| .
\end{aligned}
$$


Since $\left\{w_{\tau(n)}\right\}$ is bounded, and from the definition of $\alpha_{\tau(n)}$, we have $\lim _{n \rightarrow \infty}\left\|x_{\tau(n)+1}-x^{*}\right\|^{2}=$ 0 . This means that $\lim _{n \rightarrow \infty} a_{\tau(n)+1}=0$. It follows from (50) that $\lim _{n \rightarrow \infty} a_{n}=0$. Hence $\lim _{n \rightarrow \infty}\left\|x_{n}-x^{*}\right\|^{2}=0$. This completes the proof.

\section{Numerical examples}

Let $H=\mathbb{R}^{n}$ and $C=\left\{x \in \mathbb{R}^{n}:-5 \leq x_{i} \leq 5, \forall i \in\{1,2, \ldots, n\}\right\}$. Let the bifunction $g: \mathbb{R}^{n} \times$ $\mathbb{R}^{n} \rightarrow \mathbb{R}$ be defined by

$$
g(x, y)=\langle P x+Q y, y-x\rangle \quad \text { for all } x, y \in \mathbb{R}^{n},
$$

where $P$ and $Q$ are randomly symmetric positive semidefinite matrices such that $P-Q$ is positive semidefinite. Then $g$ is pseudomonotone on $\mathbb{R}^{n}$. Indeed, let $g(x, y) \geq 0$ for every $x, y \in \mathbb{R}^{n}$, we have

$$
\begin{aligned}
g(y, x) \leq g(x, y)+g(y, x) & =\langle P x+Q y, y-x\rangle+\langle P y+Q x, x-y\rangle \\
& =-\langle(P-Q)(x-y), x-y\rangle \leq 0 .
\end{aligned}
$$

Next, we obtain that $g$ is Lipschitz-type continuous with $L_{1}=L_{2}=\frac{1}{2}\|P-Q\|$. Indeed, for each $x, y, z \in \mathbb{R}^{n}$,

$$
\begin{aligned}
g(x, y)+g(y, z)-g(x, z) & =\langle P x+Q y, y-x\rangle+\langle P y+Q z, z-y\rangle-\langle P x+Q z, z-x\rangle \\
& =\langle(P-Q)(x-y), y-z\rangle \\
& \geq-2 \frac{\|P-Q\|}{2}\|x-y\|\|y-z\| \\
& \geq-\frac{\|P-Q\|}{2}\|x-y\|^{2}-\frac{\|P-Q\|}{2}\|y-z\|^{2},
\end{aligned}
$$

where $\|P-Q\|$ is the spectral norm of the matrix $\|P-Q\|$, that is, the square root of the largest eigenvalue of the positive semidefinite matrix $(P-Q)^{T}(P-Q)$. It is easy to check that $\Omega \neq \emptyset$. Furthermore, we define the bifunction $f: \mathbb{R}^{n} \times \mathbb{R}^{n} \rightarrow \mathbb{R}$ as

$$
f(x, y)=\langle A x+B y, y-x\rangle \quad \text { for all } x, y \in \mathbb{R}^{n},
$$

with $A$ and $B$ being positive definite matrices defined by

$$
B=N^{T} N+n I_{n} \quad \text { and } \quad A=B+M^{T} M+n I_{n}
$$

where $M, N$ are randomly $n \times n$ matrices and $I_{n}$ is the identity matrix. Then we have $f$ is $n$-strongly monotone on $\mathbb{R}^{n}$. Indeed, let $x, y \in \mathbb{R}^{n}$, we get

$$
\begin{aligned}
f(x, y)+f(y, x) & =\langle A x+B y, y-x\rangle+\langle A y+B x, x-y\rangle \\
& =-\langle(A-B)(x-y), x-y\rangle \\
& =-\left\langle M^{T} M+n I_{n}(x-y), x-y\right\rangle \\
& =-\left\langle M^{T} M(x-y), x-y\right\rangle-\left\langle n I_{n}(x-y), x-y\right\rangle
\end{aligned}
$$


Table 1 The results computed on Algorithm 1

\begin{tabular}{rlcc}
\hline$n$ & N.P. & Average iteration & Average times \\
\hline 5 & 10 & 72 & 0.7219 \\
10 & 10 & 255 & 2.3859 \\
50 & 10 & 1224 & 12.6235 \\
100 & 10 & 1541 & 37.8766 \\
500 & 10 & 1910 & 465.510 \\
\hline
\end{tabular}

$$
\begin{aligned}
& =-\|M(x-y)\|^{2}-n\|x-y\|^{2} \\
& \leq-n\|x-y\|^{2} .
\end{aligned}
$$

Moreover, $\partial_{2} f(x, x)=\{(A+B) x\}$ and $\|(A+B) x-(A+B) y\| \leq\|A+B\|\|x-y\|$ for all $x, y \in \mathbb{R}^{n}$. Thus the mapping $x \rightarrow \partial_{2} g(x, x)$ is bounded and $\|A+B\|$-Lipschitz continuous on every bounded subset of $H$. In this example, we consider the quadratic optimization

$$
\min _{x \in C}\left\{\frac{1}{2} x^{T} H x+f^{T} x\right\}
$$

where $H$ is a matrix, $f$ and $x$ are vectors. From the subproblem of solving $y_{k}$ and $z_{k}$ in Algorithm 1, we can consider problem (76).

We have tested for this example where $n=5,10,50,100$, and 500. Starting point $x_{0}$ is a randomly initial point. Take the parameters

$$
\alpha_{k}=\frac{1}{k+4}, \quad \eta_{k}=\frac{k+1}{3(k+4)}, \quad \lambda_{k}=\frac{1}{2\|P-Q\|}, \quad \mu=\frac{2}{\|A+B\|^{2}}
$$

We have implemented Algorithm 1 for this problem in Matlab R2015 running on a Desktop with Intel(R) Core(TM) i5-7200u CPU $2.50 \mathrm{GHz}$, and 4 GB RAM, and we used the stopping criteria $\left\|x_{k+1}-x_{k}\right\|<\varepsilon$ with $\varepsilon=0.001$ is a tolerance to cease the algorithm. Denote that

- N.P: the number of the tested problems.

- Average iteration: the average number of iterations.

- Average times: the average CPU-computation times (in s).

The computation results are reported in the following tables.

From the numerical result Table 1, we see that the sequence generated by our algorithms is convergent and effective for solving the solution of bilevel equilibrium problems.

\section{Conclusions}

We have proposed two iterative algorithms for finding the solution of a bilevel equilibrium problem in a real Hilbert space. The sequence generated by our algorithms converges strongly to the solution. Furthermore, we reported the numerical result to support our algorithm. 


\section{Competing interests}

The authors declare that they have no competing interests.

\section{Authors' contributions}

All authors contributed equally to this work. All authors read and approved the final manuscript.

\section{Author details}

'Department of Mathematics, Faculty of Science, Naresuan University, Phitsanulok, Thailand. ${ }^{2}$ Faculty of Information Technology, Le Quy Don Technical University, Hanoi, Vietnam. ${ }^{3}$ Department of Applied Mathematics, Pukyong National University, Busan, Korea. ${ }^{4}$ Center of Excellence in Nonlinear Analysis and Optimization, Faculty of Science, Naresuan University, Phitsanulok, Thailand.

\section{Publisher's Note}

Springer Nature remains neutral with regard to jurisdictional claims in published maps and institutional affiliations.

\section{Received: 25 July 2018 Accepted: 5 November 2018 Published online: 26 November 2018}

\section{References}

1. Anh, P.N., Anh, T.T.H., Hien, N.D.: Modified basic projection methods for a class of equilibrium problems. Numer. Algorithms https://doi.org/10.1007/s11075-017-0431-9

2. Bento, G.C., Cruz Neto, J.X., Lopes, J.O., Soares, P.A. Jr, Soubeyran, A.: Generalized proximal distances for bilevel equilibrium problems. SIAM J. Optim. 26, 810-830 (2016)

3. Bianchi, M., Schaible, S.: Generalized monotone bifunctions and equilibrium problems. J. Optim. Theory Appl. 90, 31-43 (1996)

4. Chadli, O., Chbani, Z., Riahi, H.: Equilibrium problems with generalized monotone bifunctions and applications to variational inequalities. J. Optim. Theory Appl. 105, 299-323 (2000)

5. Chbani, Z., Riahi, H.: Weak and strong convergence of proximal penalization and proximal splitting algorithms for two-level hierarchical Ky Fan minimax inequalities. Optimization 64, 1285-1303 (2015)

6. Daniele, P., Giannessi, F., Maugeri, A.: Equilibrium Problems and Variational Models. Kluwer Academic, Norwell (2003)

7. Dempe, S.: Annotated bibliography on bilevel programming and mathematical programs with equilibrium constraints. Optimization 52, 333-359 (2003)

8. Dinh, B.V., Kim, D.S.: Extragradient algorithms for equilibrium problems and symmetric generalized hybrid mappings. Optim. Lett. https://doi.org/10.1007/s11590-016-1025-5

9. Hieu, D.V.: Weak and strong convergence of subgradient extragradient methods for pseudomonotone equilibrium. Commun. Korean Math. Soc. 31, 879-893 (2016)

10. Hieu, D.V., Moudafi, A.: A barycentric projected-subgradient algorithm for equilibrium problems. J. Nonlinear Var. Anal. 1, 43-59 (2017)

11. liduka, H.: Fixed point optimization algorithm and its application to power control in CDMA data networks. Math. Program. 133, 227-242 (2012)

12. liduka, H., Yamada, I.: A use of conjugate gradient direction for the convex optimization problem over the fixed point set of a nonexpansive mapping. SIAM J. Optim. 19, 1881-1893 (2009)

13. Iusem, A.N.: On the maximal monotonicity of diagonal subdifferential operators. J. Convex Anal. 18, 489-503 (2011)

14. Jusem, A.N., Nasri, M.: Inexact proximal point methods for equilibrium problems in Banach spaces. Numer. Funct. Anal. Optim. 28, 1279-1308 (2007)

15. Kim, D.S., Dinh, B.V.: Parallel extragradient algorithms for multiple set split equilibrium problems in Hilbert spaces. Numer. Algorithms 77, 741-761 (2018)

16. Konnov, I.V.: Application of the proximal point method to nonmonotone equilibrium problems. J. Optim. Theory Appl. 119, 317-333 (2003)

17. Liu, Y: A modified hybrid method for solving variational inequality problems in Banach spaces. J. Nonlinear Funct. Anal. 2017, Article ID 31 (2017)

18. Mainge, P.E.: Strong convergence of projected subgradient methods for nonsmooth and nonstrictly convex minimization. Set-Valued Anal. 16, 899-912 (2008)

19. Moudafi, A.: Proximal point algorithm extended to equilibrium problems. J. Nat. Geom. 15, 91-100 (1999)

20. Moudafi, A.: Proximal methods for a class of bilevel monotone equilibrium problems. J. Glob. Optim. 47, 287-292 (2010)

21. Muu, L.D., Oettli, W.: Optimization over equilibrium sets. Optimization 49, 179-189 (2000)

22. Muu, L.D., Quoc, T.D.: Regularization algorithms for solving monotone Ky Fan inequalities with application to a Nash-Cournot equilibrium model. J. Optim. Theory Appl. 142, 185-204 (2009)

23. Quy, N.V.: An algorithm for a bilevel problem with equilibrium and fixed point constraints. Optimization $64,1-17$ (2014)

24. Thuy, L.Q., Hai, T.N.: A projected subgradient algorithm for bilevel equilibrium problems and applications. J. Optim. Theory Appl. https://doi.org/10.1007/s10957-017-1176-2

25. Tran, D.Q., Muu, L.D., Nguyen, V.H.: Extragradient algorithms extended to equilibrium problems. Optimization 57, 749-776 (2008)

26. Vuong, P.T., Strodiot, J.J., Nguyen, V.H.: Extragradient methods and linesearch algorithms for solving Ky Fan inequalities and fixed point problems. J. Optim. Theory Appl. 155, 605-627 (2013)

27. Xu, H.K.: Iterative algorithms for nonlinear operators. J. Lond. Math. Soc. 66, 240-256 (2002)

28. Yao, Y., Petrusel, A., Qin, X.: An improved algorithm based on Korpelevich's method for variational inequalities in Banach spaces. J. Nonlinear Convex Anal. 19, 397-406 (2018) 